\title{
Well spotted: but now you need to do something
}

\author{
Richard Hamblin, Carl Shuker
}

Health Quality Intelligence, New Zealand Health Quality and Safety Commission, Wellington, New Zealand

\section{Correspondence to} Mr Richard Hamblin, New Zealand Health Quality and Safety Commission, Wellington, New Zealand;

richard.hamblin@hqsc.govt.nz

Accepted 3 September 2020 Published Online First 18 September 2020

\section{SLinked}

- http://dx.doi.org/10.1136/ bmjqs-2019-010141

\section{Check for updates}

(c) Author(s) (or their employer(s)) 2021. No commercial re-use. See rights and permissions. Published by BMJ.

To cite: Hamblin R, Shuker C. BMJ Qual Saf

2021:30:177-179.
The ability to spot services at risk of becoming unsafe, in order to address the underpinning causes and avoid the sorts of failures seen in Mid Staffordshire, ${ }^{1}$ Walter Reed ${ }^{2}$ or King Edward ${ }^{3}$ has become an increasing preoccupation of health systems in the developed world. In this issue, Nowotny and colleagues contribute to the emerging knowledge in this area with their retrospective study of which routinely available data may have given early warning of a service failure in a maternity service in Victoria, Australia. ${ }^{4}$

To date, most national approaches to using routine data to identify poor quality have concentrated on posthoc analyses of sentinel poor outcomes-typically clusters of deaths from similar diagnoses within hospitals and units. ${ }^{56}$ Done well, with appropriate statistical sophistication, this can allow relatively quick identification of emerging problems. Indeed, the Mid Staffordshire case was first spotted using precisely this approach. ${ }^{7}$

The approach adopted by Nowotny and colleagues moves one step beyond the monitoring of sentinel outcomes, triangulating a range of data pointing in the same direction and identifying which have predictive power in this case. They note that rapidly increasing clinical activity (or pressure on services) was associated with safety failure. This is worrying in the current environment. Even in many countries that appear to have gained control of the COVID-19 pandemic, a 'backlog' of patients who did not access services at the height of the pandemic has occurred. At the same time as this likely increase in demand, the need to keep a 'corridor' for patients with COVID-19 separated from others further reduces supply. Increased pressure on services is a likely fact of life in nearly all health systems over the next few years. In this context, Nowotny and colleagues' results are therefore timely and urgent.
However, although the ability to interpret historic outcomes to predict future risk is essential, two further developments are required to marry data to safety.

First, we need to recognise that to rely solely on historic data to predict future risk is rather akin to attempting to 'drive by looking in the rear-view mirror'. An alternative is to consider a broader range of information about organisational culture, resilience and reliability and ability to respond to new risks.

In their framework for safety measurement and monitoring, ${ }^{8-10}$ Vincent and colleagues suggest five dimensions that need to be measured to understand how safe a healthcare organisation is. Only one of these, past harm, can be measured through either sentinel events data or triangulating use of outcome data. The others-reliability; sensitivity to operations; anticipation and preparedness and integration and learning-speak much more to an organisation's systems and processes, culture and capacity. Weaknesses in these areas may be an 'earlier warning' of problems ahead than even the most refined posthoc analysis of historic poor outcomes. Getting hold of data (in the broad sense of the word) to understand performance in these four 'present tense' dimensions, may be the challenge.

Resilient healthcare, in the framing of Braithwaite and colleagues, recognises that the current approaches to poor safety performance in many healthcare systems use a linear 'find and fix' model, ${ }^{1}$ that is, root cause analysis of the $10 \%$ or $5 \%$ or $1 \%$ of adverse outcomes, identification of the issue, error or individual at the 'root', and response, whether punitive or through regulation or policy, to minimise future occurrences. The paradigm shift Braithwaite and colleagues advocate instead is a focus on 'Safety-II'-a recognition of the complexity of the system within which errors and failures occur, 
and the concomitant fostering and nurturing of the conditions under which things go right, as they more often do, rather than go wrong.

Such an acknowledgement and such an approach are predicated on the idea that the complex emergent environments of healthcare delivery require the system's ability to rapidly recognise and adjust to changing contexts. COVID-19, in many jurisdictions, is the signal example, where command-and-control approaches under tremendous pressure ceded power to local responses with more distributed leadership and devolved decision-making in an atmosphere of trust in local actors to work toward overarching goals. When conditions are dynamic, complex and uncertain (all of the time in healthcare, even more so during a pandemic), the ability to recognise, understand and adjust is crucial.

The second development required is knowing how to react to a warning sign. Reviews of service failures tend to note examples of where evidence of failure was available but unobserved and where evidence of failure was observed but not acted on. This raises the question: who should act, and how? One of the ironies of health services in developed countries over the last 30 years is that while data about operation of services has proliferated, systems have tended to artificially limit their options to respond to these signals. Typically, regulatory and performance management mechanisms have been privileged over other alternatives such as quality improvement, training and education, or policy and resourcing changes.

This seems short-sighted. At the heart of consideration of poor quality care is the paradox that while studies commonly estimate that in the order of 1 in 10 hospital patients are in some way harmed by the care they receive, ${ }^{11}$ the sorts of scandals mentioned above shock, in part, because of their rarity. They are not 'normal' (indeed, perverse normalisation of shockingly poor care is part of what causes these rare scandals. ${ }^{12}$ In other words, there is a danger of only concentrating on the notorious scandals while missing the slow accretion of suboptimal care (and especially systems of care with in-built inequity), which at the population level is responsible for more damage.

Yet regulatory and performance management responses are designed to do precisely this-to address the malicious, reckless or incompetent professional or the 'failing organisation', even though these are only comparatively rarely the cause of poor quality or unsafe care. A broader range of causes need to be considered, as we propose in the provisional description shown in figure 1. As well as the underpinning social determinants of health, poor outcomes at a triple/quadruple aim level can be driven by a range of causes. ${ }^{13}$ As such, a range of responses are required, including, always, engagement and partnership with consumers in support. Along with the 'bad apple' and the 'failing organisation', problems may reflect:

- teams that lack capability (such as knowledge, skills or well-designed processes).

- organisations that are wrongly designed or resourced for the task they have to do (indeed Nowotny and colleagues' identification of activity per bed points to this as a common cause).

- the whole health system may be poorly designed (at least for the specific service under consideration).

- a risk emerging from a new and insufficient tested therapy (surgical mesh in gynaecological patients is a recent example ${ }^{14}$ ).

For these different causes, different approaches may be required, as the figure suggests. These certainly include regulation and performance management, but

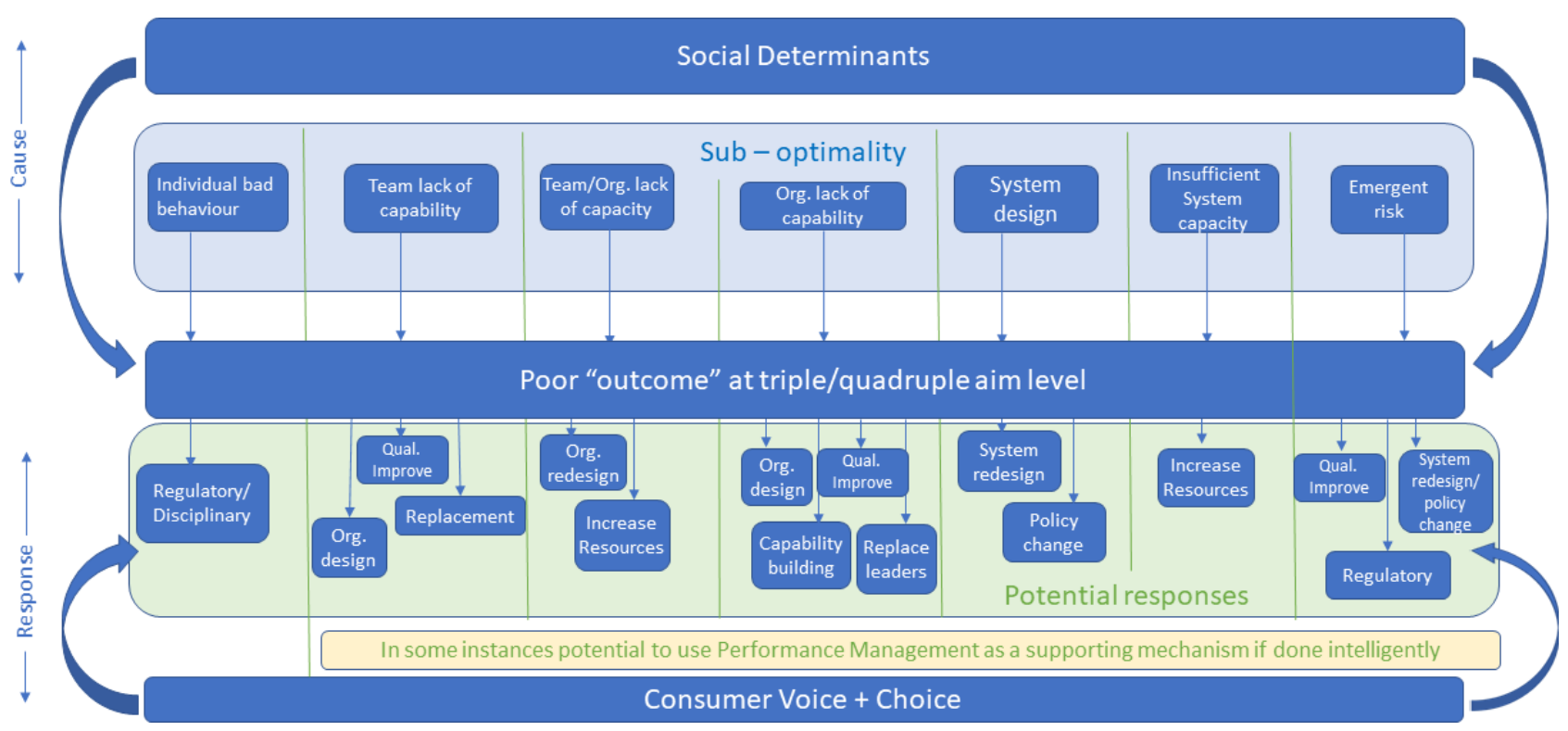

Figure 1 Why things go wrong and how to address them. 
also encompass quality improvement science, capability building at the team or organisational level, enhanced skills in clinical governance, policy change and in some cases simply more resources. It may also be necessary to consider reconfiguration of services and changes of leadership, although recent evidence suggesting that organisational instability is linked with poorer performance ${ }^{15}$ suggests that these options should be closer to a last resort than a first recourse.

What this implies is a need for health systems to have mechanisms for understanding the cause of potential poor quality that are as rigorous as their systems for spotting its likelihood. Building this capability has several prerequisites. Concepts such as complex adaptive systems (and the recognition that this is what health systems are), ${ }^{16}$ resilient organisations ${ }^{17}$ and human factors ${ }^{18}$ need to be much more widely understood.

Above all, trust between frontline services and central system governance is essential. Building the analytic capability to understand the sorts of variants of statistical process control required to have early warning of poor-quality care is a prerequisite to making such a system work, but without trust these skills can (and have) simply become weaponised to argue the toss about whether there is a problem or not. When this happens, patients lose.

Funding The authors have not declared a specific grant for this research from any funding agency in the public, commercial or not-for-profit sectors.

Competing interests None declared.

Patient consent for publication Not required.

Provenance and peer review Not commissioned; internally peer reviewed.

\section{REFERENCES}

1 Braithwaite J, Wears RL, Hollnagel E. Resilient health care: turning patient safety on its head. Int J Qual Health Care 2015;27:418-20.

2 Blum N, Fee E, Dee E. Critical shortcomings at Walter Reed Army medical center create doubt. Am J Public Health 2008;98:2159-60.

3 Safety and Quality Council. Lessons from the inquiry into obstetrics and gynaecological services at King Edward Memorial Hospital 1990-2000. Available: https://www. safetyandquality.gov.au/sites/default/files/migrated/king_ edward.pdf [Accessed 21 Aug 2020].

4 Nowotny BM, Davies-Tuck M, Scott B, et al. Preventing critical failure. Can routinely collected data be repurposed to predict avoidable patient harm? A quantitative descriptive study. BMJ Qual Saf 2021;30:186-94.

5 Spiegelhalter D, Sherlaw-Johnson C, Bardsley M, et al. Statistical methods for healthcare regulation: rating, screening and surveillance. J R Stat Soc Ser A Stat Soc 2012;175:1-47.

6 Coory M, Duckett S, Sketcher-Baker K. Using control charts to monitor quality of hospital care with administrative data. Int J Qual Health Care 2008;20:31-9.

7 Wood H. Witness statement to the mid Staffordshire NHS Foundation trust public inquiry. exhibit HW1=HW19. 18 April 2011. The mid Staffordshire NHS Foundation trust public inquiry. available at. Available: https://webarchive. nationalarchives.gov.uk/20150407085448/http://www.midstaff spublicinquiry.com/hearings/s/313/week-twenty-two-9-12-may2011 [Accessed 22 Aug 2020].

8 Vincent C, Burnett S, Carthey J. Safety measurement and monitoring in healthcare: a framework to guide clinical teams and healthcare organisations in maintaining safety. BMJ Qual Saf 2014;23:670-7.

9 Vincent C, Burnett S, Carthey J. The measurement and monitoring of safety. The health Foundation, London, 2013. Available: https://www.health.org.uk/publications/ the-measurement-and-monitoring-of-safety [Accessed 25 Aug 2020].

10 Braithwaite J, Wears RL, Hollnagel E. Resilient health care: turning patient safety on its head. Int J Qual Health Care 2015;27:418-20.

11 Davis P, Lay-Yee R, Briant R, et al. Adverse events in New Zealand public hospitals I: occurrence and impact. N Z Med J 2002;115:U271.

12 Hendy J, Tucker DA. Public sector organizational failure: a study of collective denial in the UK National health service. J Bus Ethics 2020 doi:10.1007/s10551-020-04517-1. [Epub ahead of print: 30 Apr 2020].

13 Sikka R, Morath JM, Leape L. The quadruple aim: care, health, cost and meaning in work. BMJ Qual Saf 2015;24:608-10.

14 Haskell H. Cumberlege review exposes stubborn and dangerous flaws in healthcare. BMJ 2020;370:m3099.

15 Wynen J, Verhoest K, Kleizen B. Are public organizations suffering from repetitive change injury? A panel study of the damaging effect of intense reform sequences. Governance 2019;32:695-713.

16 Begun JW, Zimmerman B, Dooley K. Health Care Organizations as Complex Adaptive Systems. In: Mick SM, Wyttenbach M, eds. Advances in health care organization theory. 1st ed. San Francisco, California, USA: Jossey-Bass, 2003Pp.: 253-88.

17 Beer M. High commitment high performance: how to build a resilient organization for sustained advantage. Wiley: Hoboken NJ, 2009.

18 Reason J. Understanding adverse events: human factors. Qual Health Care 1995;4:80-9. 